\title{
Gradhiva
}

GRADHIV

Revue d'anthropologie et d'histoire des arts

$11 \mid 2010$

Grands hommes vus d'en bas

\section{Du Golgotha au tzompantli}

Les crânes « aztèques » en cristal de roche

From Golgotha to tzompantli: the "Aztec" crystal skulls

\section{Pascal Mongne}

\section{CpenEdition}

Journals

Édition électronique

URL : http://journals.openedition.org/gradhiva/1734

DOI : 10.4000/gradhiva. 1734

ISSN : 1760-849X

Éditeur

Musée du quai Branly Jacques Chirac

Édition imprimée

Date de publication : 19 mai 2010

Pagination : 181-187

ISBN : 978-2-35744-025-8

ISSN : 0764-8928

Référence électronique

Pascal Mongne, « Du Golgotha au tzompantli », Gradhiva [En ligne], 11 | 2010, mis en ligne le 19 mai 2013, consulté le 21 avril 2019. URL : http://journals.openedition.org/gradhiva/1734 ; DOI : 10.4000/ gradhiva.1734

(c) musée du quai Branly 


\section{Du Golgotha au tzompantli Les crânes «aztèques" en cristal de roche}

Pascal Mongne

Une récente production cinématographique à grand spectacle, dont le héros est un archéologue aux méthodes parfois expéditives, vient de placer sous les feux de l'actualité un type d'objet particulier, traditionnellement attribué à la civilisation aztèque : les crânes de cristal de roche. Ce soudain regain d'intérêt intervient alors que depuis plusieurs années la communauté scientifique remet en cause l'authenticité de ces objets. La nature particulière de ces pièces ainsi que l'aura de mystère et de tromperie qui les entoure appellent quelques réflexions, ici exposées.

Plusieurs dizaines de crânes réalisés en cristal de roche, attribués à la culture aztèque, sont aujourd'hui comptés dans le monde. Deux groupes peuvent être distingués : les "petits » crânes, dont les dimensions varient entre 2 et $6 \mathrm{~cm}$, et dont le nombre exact n'est pas connu; les "grands » crânes, proches de la grandeur nature $(10$ à $25 \mathrm{~cm}$ ) et beaucoup moins nombreux (une douzaine recensés dans le monde). Par leur taille, ces derniers sont évidemment les mieux connus, notamment ceux du National Museum of Natural History de Washington, du British Museum de Londres et bien sûr du musée du quai Branly à Paris (López Luján et Fauvet-Berthelot 2005).

Ces objets, aujourd'hui célèbres, sont les parfaits reflets de l'image morbide du monde aztèque véhiculée par l'Occident, et notamment de ses spectaculaires et fort nombreux sacrifices humains. Par leurs aspects et par la cavité cylindrique qui les perce, ils ont été associés dès leur apparition, durant la seconde moitié du xix ${ }^{e}$ siècle, aux rituels des tzompantli, plateformes sur lesquelles des milliers de crânes de sacrifiés étaient exposés dans l'enceinte sacrée de Tenochtitlán, la capitale des Aztèques. Réalisés en cristal de roche, de belle facture et d'un grand réalisme, ils sont traditionnellement considérés comme de parfaits exemples du savoir-faire lapidaire aztèque. "Apprécié, précieux et vénéré », comme le signale Bernardino de Sahagún, ce matériau fut en effet l'objet d'un artisanat de grande qualité : bijoux, labrets, ornements d'oreilles, etc. 
Fig. 1 Représentation de crâne humain en cristal de roche, Mexico @ musée du quai Branly, photo Patrick Gries, Valérie Torre.
Cependant, sachons que les grands crânes n'apparaissent pas au nombre des objets connus pour avoir été réalisés à l'époque aztèque (aucun n'est issu d'un contexte archéologique), et que des analyses physicochimiques menées d'abord à Londres et à Washington (Sax et alii 2008) puis à Paris (Calligaro et alii 2009) signalent l'origine non mexicaine d'un matériau travaillé avec des instruments d'orfèvrerie européenne. De tels résultats entretiennent - on s'en doute- de sérieux soupçons sur l'authenticité de ces crânes; soupçons renforcés auprès de Jane MacLaren Walsh qui, depuis plusieurs années, en étudie l'histoire muséographique. Le chercheur de la Smithsonian Institution démontre en effet que plusieurs d'entre eux proviennent d'une même source, la collection d'Eugène Boban (MacLaren Walsh 1997, 2005, 2008). Cet antiquaire français, célèbre en son temps, fut l'un des principaux fournisseurs d'art précolombien auprès des collectionneurs européens et nord-américains (Riviale 2001).

En somme, et au vu des analyses et des recherches menées jusqu'alors, il apparaît que plusieurs des grands crânes (sinon tous?) auraient pu être taillés durant le $\mathrm{xIX}^{\mathrm{e}}$ siècle en Europe germanique (principalement à Idar-Oberstein, grand centre d'art lapidaire depuis le $\mathrm{xv}^{\mathrm{e}}$ siècle) à partir d'un cristal de roche brésilien, lequel fut semble-t-il importé en masse grâce à une importante diaspora d'artisans allemands émigrés au Brésil (certaines sources citent également une origine malgache du matériau). Arrivés au Mexique, ces objets auraient été ainsi, selon MacLaren Walsh, l'enjeu d'une opération de tromperie dont l'antiquaire Boban serait le principal commanditaire. Ces très intéressants résultats concluent donc à l'existence d'une des plus importantes affaires de faux que l'art précolombien ait connues.

S'il n'est pas question de développer ici une réflexion sur la question du faux en art, plusieurs notions doivent cependant être abordées car indispensables à la compréhension du terme même et de ce qu'il recouvre. En fait, et pour résumer les recherches menées dans ce domaine depuis longtemps (Chamoux et alii 1973; Kurz 1967; Teitze 1934-1936), trois règles président à l'apparition du faux artistique :

- Le faux en art "n'existe pas». Cet axiome volontiers provocant s'appuie cependant sur une constatation juridique simple : il n'y a pas de fausse œuvre d'art "comme le serait un kilo de beurre laitier fabriqué avec des huiles végétales... " (Kiejman 1973 : 126). En effet, quels que soient son domaine, sa source, son âge, une œuvre d'art ne verra jamais sa nature changer après qu'elle a été dénoncée comme fausse. Ses caractéristiques ne se volatiliseront pas brutalement devant ses juges : seule en fait sa valeur financière aura fondu. Le faux en art n'existe donc pas par nature, mais par intention...

- Le faux en art est une "créature" du marché de l'art. Bien que les causes du faux artistique puissent être plurielles (religieuses, scientifiques, psychologiques, canulars, etc.), celles-là sont cependant fort rares. Les principales raisons de son existence sont en fait essentiellement commerciales : le faux étant principalement issu d'une demande supérieure à l'offre, demande attisée par la spéculation sur certains domaines du marché de l'art. Membre de la sinistre triade qui en résulte - avec le pillage et le trafic des œuvres -, il en est paradoxalement le contrepoids, le parasite "salutaire », en admettant qu'il se substitue au Vrai et provoque, par les accès de fièvre médiatique dont on l'entoure, l'inhibition du marché de l'art.

- Le faux en art est "l'enfant de son temps». Produit à des époques et dans des milieux variés, il est le reflet fidèle des goûts de la société qui le voit naître. Reflet des préférences du collectionneur qui le commande, des tendances mercantiles et des connaissances scientifiques du moment, il est enfin et surtout l'image " caricaturée » du vrai supposé à son époque de création. 


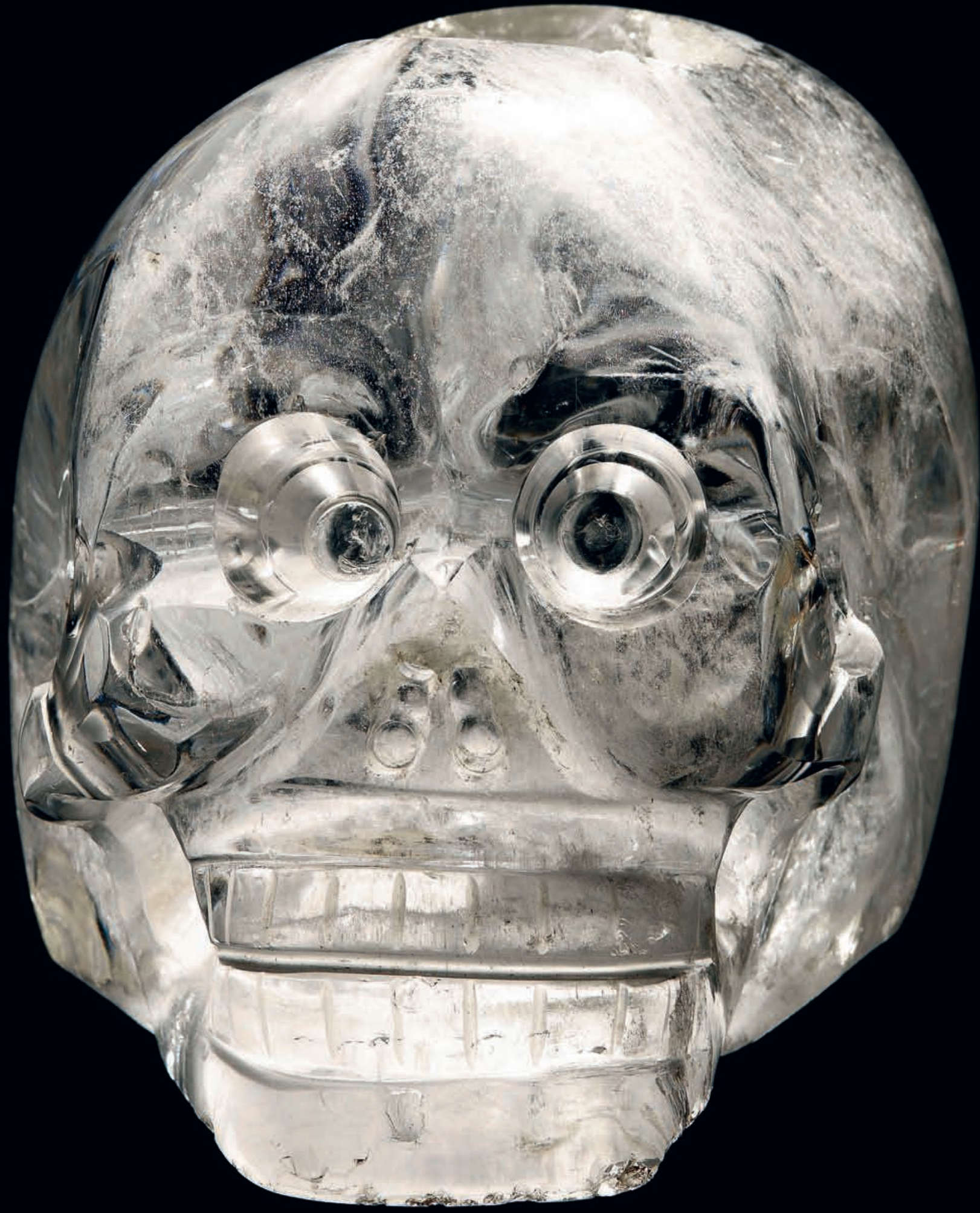


Le faux en art précolombien n'échappant pas à ces règles (Mongne 2000, 2009), plusieurs remarques s'imposent alors qui, sans aucunement remettre en cause les importants résultats apportés par MacLaren Walsh, peuvent contribuer à nuancer la traditionnelle dichotomie en la matière (faux ou vrai).

Tout d'abord, relativement à la question de la rentabilité : minéral utilisé depuis fort longtemps en bijouterie et en art décoratif, le cristal de roche est une variété de quartz connue pour ses qualités de transparence et de réfringence, et pour sa grande dureté (7 sur l'échelle de Moss qui possède 10 degrés). Cette dernière propriété le rend difficile à travailler, et implique inévitablement la dextérité d'un artisan professionnel et l'utilisation d'outillage performant. Un travail long, précautionneux et coûteux en est donc la conséquence, auquel doit être ajouté le prix de revient d'une matière qui, si l'on s'en tient aux récentes découvertes, aurait traversé deux fois l'Atlantique. Des questions se posent alors : quel intérêt aurait eu un faussaire (ou son commanditaire) dans la réalisation d'une pièce d'une telle qualité, donc chère et probablement difficilement négociable, alors que l'espoir d'une rentabilité confortable et si possible rapide est la condition même de l'existence du faux? Quel intérêt aurait eu ce même "créateur » dans la réalisation d'une telle pièce, alors que l'obtention et la vente d'objets authentiques, aisément accessibles malgré les lois en vigueur de l'époque, autorisaient des bénéfices non négligeables?

Ensuite, relativement à la question du goût et des modes : si le faux est «l'enfant de son temps ", ces crânes, d'une grande qualité esthétique, réalistes et proches des canons reconnus de l'art aztèque, ne pouvaient correspondre à l'idée que l'on avait de cet art à la fin du xix ${ }^{e}$ siècle. Engoncé dans un ethnocentrisme triomphant, la Vieille Europe (et donc les États-Unis) ne pouvait considérer à sa véritable valeur les arts précolombiens. Voyant dans les réalisations matérielles indigènes, passées ou présentes, non une forme d'art mais de simples productions non civilisées, indignes des grands musées d'art (d'où la création contemporaine de musées «d'ethnographie»), cet ethnocentrisme développe tout naturellement une vision négative dont le faux est inévitablement le reflet. La réalisation de contrefaçons en céramique (figurines et poteries) de Teotihuacán et Tlatelolco, apparue durant la seconde moitié du xix siècle (Holmes 1886), est à ce titre édifiante, qui mêle aux formes européennes (cruches, jarres) les pseudo-canons indiens : laideur, difformité, sauvagerie. Il est alors difficile d'imaginer une production de qualité et surtout valorisante, dont le but - rappelons-le - était de plaire à une clientèle marquée par son époque.

Enfin, relativement au personnage controversé d'Eugène Boban. C'est au cours de son long séjour à Mexico (des années 1850 à 1869, puis de 1884 à 1886) que Boban s'est forgé une solide réputation d'antiquaire. Il était ainsi devenu l'un des principaux fournisseurs en art précolombien, non seulement sur les places de Mexico et de Paris, mais aussi aux États-Unis. Ayant noué dès les débuts de son activité des liens étroits avec les spécialistes d'un américanisme balbutiant, il était devenu lui-même un grand connaisseur et l'auteur de plusieurs catalogues et publications d'intérêt (Riviale 2001). Aussi, il peut sembler étrange que ce personnage honorablement connu, ayant pignon sur rue, se soit engagé dans une telle opération de contrefaçon dont les risques étaient loin d'être négligeables, notamment pour sa réputation. En outre, quel avantage aurait-il pu tirer de la fabrication d'objets coûteux, dont la vente n'était après tout point assurée, alors que les pièces authentiques, aisées à trouver par son réseau, alimentaient déjà une clientèle fournie, riche et fidèle? Certes, on ne peut ignorer que rôdent autour de lui de sombres rumeurs de faux, dont celle d'un crâne de cristal de roche, cause 
de son départ définitif du Mexique en 1886 selon MacLaren Walsh. En serait-il cependant l'auteur, ou même le complice? Bien que plusieurs de ces objets soient passés entre ses mains, rien ne permet d'affirmer qu'il fut le commanditaire de leur fabrication. Ni même qu'il eut conscience de leur nature, à une époque où les collections publiques et privées semblaient regorger de faux (Holmes 1886).

$\mathrm{Si}$, au vu des informations rassemblées ces dernières années, les spécialistes s'accordent désormais à considérer les grands crânes comme de fabrication «récente", portant les marques des techniques lapidaires européennes et de surcroît réalisés dans une matière non originaire du Mexique, il semble en revanche beaucoup plus délicat d'y voir - pour les raisons exposées plus haut - des contrefaçons, donc réalisées dans l'intention de tromper. En revanche, la question d'une attribution erronée peut être abordée. Ce type de phénomène, fort courant dans l'histoire mondiale des arts depuis la seconde moitié du xix siècle, voue aux gémonies des milliers d'objets n'ayant pas eu l'heur d'être "reconnus». Répliques, copies, imitations, pastiches, pourtant « authentiques sans être originaux » (Chamoux et alii 1973: 5), en sont les premières victimes. Cela peut être aussi le cas de pièces dont la fonction originelle est ignorée ou bien oubliée, et qui par leurs caractéristiques esthétiques se trouvent hissées aux sommets des intérêts scientifiques et mercantiles. Sommets desquels elles chuteront à la dénonciation des incohérences iconographiques ou techniques.

La présence d'un trou vertical traversant le crâne de Paris, ainsi que la presque totalité des crânes de petite taille, a souvent été invoquée comme preuve d'une incompréhension de faussaire, voulant imiter la trace du passage du pieu maintenant le crâne sur le tzompantli. En effet, les crânes véritables trouvés en fouilles présentent un percement horizontal et non vertical : les crânes étaient enfilés et non empalés sur les pieux (sachons cependant que certaines régions de Mésoamérique pratiquèrent l'empalement des crânes - López Luján, communication personnelle). Ce point ne fait que renforcer une hypothèse (déjà envisagée par MacLaren Walsh et d'autres chercheurs) : celle d'un objet du culte catholique. Il semble en effet bien plus probable que ces cavités soient la marque d'une mortaise ayant permis la fixation d'une croix. Rien n'interdit d'imaginer que les petits crânes de cristal de roche aient été (sinon tous, du moins un bon nombre) des objets coloniaux destinés à servir de socle à des crucifix de petite taille (une trentaine de centimètres). À ce sujet, signalons la très belle croix reliquaire mexicaine de la collection Redo, datée de la fin du xvi e siècle et présentée au Metropolitan Museum de New York en 1990 (in Mexico, splendors of thirty centuries). Le crâne en cristal de roche qu'elle surmonte illustrera aisément notre propos.

Est-il alors illégitime de penser que les grands crânes - objets de toutes les spéculations - aient eu la même fonction, malgré la date tardive de fabrication qu'on attribue à ceux qui ont été analysés? Leurs dimensions les réservant, pourquoi pas, aux crucifix d'autels des églises et des chapelles. Un seul, cependant (celui de Paris), présente la cavité autorisant cette utilisation. Quelques-uns disposent d'une cavité à la base. Peut-on alors admettre que ces derniers, fichés ou sertis, aient eu une fonction associée au Golgotha, tel qu'il est traditionnellement représenté dans l'iconographie sacrée? Si c'est le cas, il fait peu de doute que ces crânes (petits ou grands), comme tant de mobiliers et lieux de culte, durent subir les effets de l'anarchie des années suivant l'indépendance du Mexique et de l'affaiblissement temporel de l'Église. Symboles religieux par excellence, les crucifix furent probablement les cibles principales des destructions anticléricales de la période de la Réforme (1855-1862) : vandalisés, détruits, au mieux vendus puis

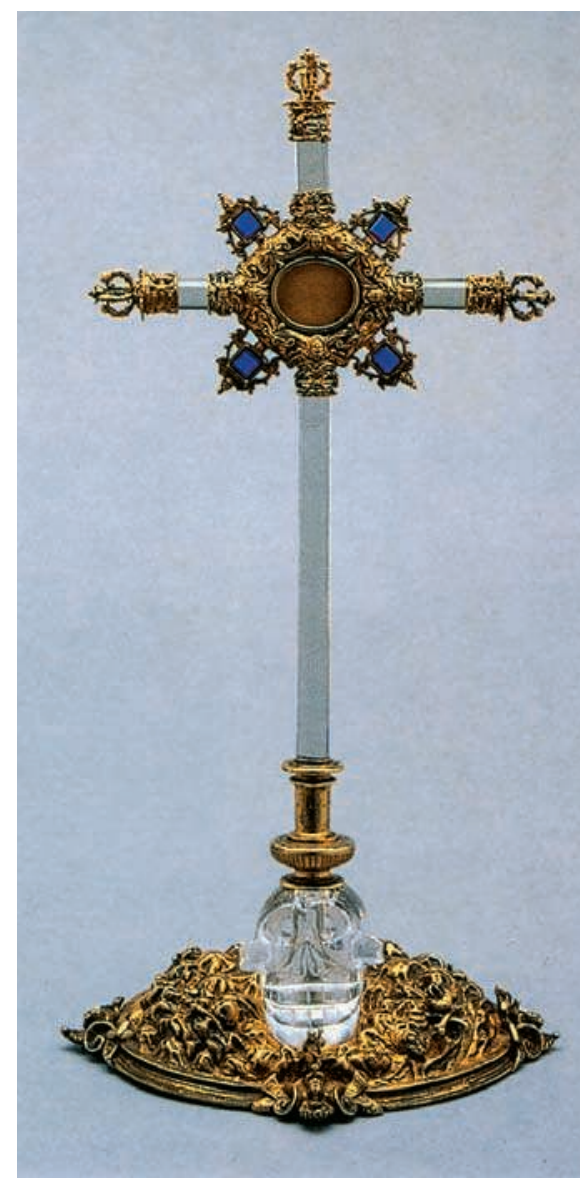

Fig. 2 Crucifix de la collection Redo, argent doré, cristal de roche, pierres bleues, sceau de cire représentant l'Agnus Dei, $41 \mathrm{~cm}$. Mexico, 1575-1578. Tirée de Mexico : splendors of Thirty centuries, 1990. The Metropolitan Museum of Art, Boston, Toronto, London, Bulfinch Press, cop. 
démantelés. Il ne serait donc pas illogique que ces objets finement taillés, redécouverts quelques décennies plus tard et libres de toute référence chrétienne, aient été investis d'une nouvelle identité et de nouvelles fonctions, à l'aune des connaissances que le siècle finissant rassemblait.

On ne peut ignorer cependant le cas de quelques autres, notamment des crânes Mitchell-Hedges (apparu dans les années 1930, pratiquement identique à celui du British Museum mais réalisé en deux parties : boîte crânienne et mandibule) et de la Smithsonian Institution (apparu dans les années 1960, creux, stylistiquement très dépouillé et d'une taille supérieure à tous les autres : $25 \mathrm{~cm}$ ). Les traces d'outillage occidental trouvées sur ces deux pièces, et même celles d'un abrasif non utilisé avant le $x^{e}$ siècle (pour le crâne de la Smithsonian), ne permettent aucun doute sur leur fabrication récente. De plus, leurs caractères physiques (réalisme et mandibule séparée pour l'un, dimensions et sobriété stylistique pour l'autre), et surtout leur date d'apparition en font des pièces particulières. Non "associables " aux fonctions religieuses évoquées, ces pièces correspondent en revanche complètement à leur époque d'apparition, la première moitié $d u x^{e}$ siècle, fortement marquée par les courants primitivistes qui rendirent aux arts exotiques leurs lettres de noblesse et inévitablement attirèrent sur eux l'intérêt du marché de l'art. "Enfants de leur époque ", "créatures du marché de l'art ", ces crânes-là (et peut-être quelques autres) peuvent aisément être considérés comme des contrefaçons.

En somme, au regard de ces quelques lignes, et en remaniant la classification de Jane MacLaren Walsh (2008), trois « générations » de crânes pourraient être distinguées. La première est celle des petits crânes, créés durant la période coloniale. La deuxième est celle des grands crânes de Paris et de Londres, fabriqués au xix siècle (le crâne de Paris pourrait être plus ancien). On peut imaginer que les pièces de la première et deuxième générations, créées pour servir de base à des crucifix, ont été démantelées pendant les décennies troublées du milieu du xix siècle, puis oubliées. Redécouverts plus tard, ces crânes auraient alors été revêtus d'une identité "aztèque ». La troisième génération inclurait les crânes apparus après la première guerre mondiale (crâne Mitchell-Hedges par exemple). Réalisées à une époque de redécouverte de l'art précolombien pour être vendues comme aztèques (en imitation des pièces précédentes), ces dernières pièces sont de «véritables » faux.

Ainsi peut être appréciée la spectaculaire évolution d'une production artistique qui, au rythme de l'histoire du goût, a connu et subi bien des avatars. Primitivement et probablement coloniale (voire plus récente) et créée pour les besoins de l'iconographie catholique, démantelée puis oubliée dans les affres des révolutions, redécouverte ensuite et réinterprétée à l'aune de l'américanisme de la fin du xix siècle, elle complète enfin son cycle de transformation par des imitations ou des contrefaçons dont les modèles n'ont jamais été précolombiens... Aussi, et si l'on excepte les pièces les plus récentes, nous pouvons admettre que les crânes aztèques en cristal de roche, dans leur grande majorité, ne peuvent être des faux et n'ont probablement jamais été fabriqués pour tromper. Ils sont en revanche les témoins actifs de l'histoire des sciences, des modes du marché de l'art et du phénomène de balancier qui anime l'image des Amériques depuis maintenant cinq siècles dans la Vieille Europe. Par son effet, le Golgotha est devenu le tzompantli.

École du Louvre / pascal.mongne@orange.fr

mots clés / keywords : attributionnisme // attributionnism • aztèque // Aztec • catholique // Catholic • colonial // colonial $\cdot$ crâne // skull $\cdot$ cristal // crystal $\cdot$ faux //fake. 


\section{Bibliographie}

Calligaro, Thomas, Coouinot, Yvan, Reiche, Ina, CAstaing, Jacques, Salomon, Joseph, Ferrand, Gérard et Le Fur, Yves

2009 « Dating study of two rock crystal carvings by surface microtopography and by ion beam analyses of hydrogen », Applied Physics A: Materials Science \& Processing $94(4)$ : 871-878.

Chamoux, François, Velmans, Tania, Grodecki, Louis, GaUtier, MarieMadeleine, SchNAPPER, Antoine, FoucART, Bruno et CHASTEL, André 1973 «Copies, répliques et faux» (éditorial), Revue de l'art 21 : 55-31.

\section{Holmes, Williams}

1886 « On some spurious Mexican antiquities and their relation to ancient art », Annual Report of the board of the regents of the Smithsonian Institution : 319-334.

\section{KiejMAN, Georges}

1973 « Le faux en art et sa répression par le droit français », Revue de l'art 21 : 125-128.

\section{KuRz, Otto}

1967 Fakes. New York, Dover Books.

LóPEZ LUJÁn Leonardo et FAUVET-

Berthelot, Marie-France

2005 Aztèques : la collection de sculptures du musée du quai Branly, Paris, musée du quai Branly.

\section{MacLaren Walsh, Jane}

1997 «Crystal skulls and other problems:

or don't look it in the eye », in Amy Henderson

et Adrienne Kaeppler, Issues of representation

at the Smithsonian Institution.

New York, Smithsonian Institution.

2005 « What is real? A new look at

Precolumbian Mesoamerican collections », AnthroNotes 26[1] : 1-7, 17-19.

2008 « Legend of the Crystal Skulls », Archaeology 61(3) : 36-41.

\section{MeXICO: SPLENDORS OF THIRTY CENTURIES}

1990 The Metropolitan Museum of Art, New York (catalogue). Boston, Bulfinch Press.

Mongne, Pascal

2000 « Le faux zapotèque et la collection
Gustave Bellon. Iconographie, thermoluminescence et nouvelles considérations 》, Techné : 53-64.

2009 Le Miroir déformant des Amériques : répliques, pastiches et faux en art précolombien. Le cas mexicain [communication présentée au $52^{e}$ Congrès international des américanistes de Séville, juillet 2006], Baessler-Archiv 56 : 125-145.

\section{Riviale, Pascal}

2001 « Eugène Boban ou les aventures d'un antiquaire au pays des américanistes », Journal de la Société des américanistes 87 : 351-362.

SAX, Margaret, WALSH, Jane M., FreEstone, Ian C., RANKIN, Andrew H. et MeEks, Nigel D.

2008 « The origin of two purportedly preColumbian Mexican crystal skulls », Journal of Archaeological Science 35(10) : 2751-2760.

Teitze, Hans

1934-1936 « The psychology and aestetic of forgery art », Metropolitan Museum Studies $5: 1-19$.

\section{Résumé / Abstract}

Pascal Mongne, Du Golgotha au tzompantli. Les crânes «aztèques » en cristal de roche - Plusieurs dizaines de crânes en cristal de roche, attribués aux Aztèques, sont comptés dans le monde, dont une douzaine de «grands» modèles. Ces derniers, par les pouvoirs «eschatologiques » que certains leur accordent, sont devenus célèbres et ont pu inspirer une récente production cinématographique. nouvelles études ont cependant démontré que ces belles pièces ne sont pas aztèques et ont été réalisées tardivement dans un matériau non mexicain. Si l'authenticité des crânes de cristal est mise en cause, doit-on les considérer tous comme des faux, destinés donc à tromper ? La question d'une attribution erronée se doit d'être abordée et permet de considérer qu'ils furent, pour la plupart, des objets de culte catholique, bases de crucifix, démantelés, oubliés puis redécouverts et enfin revêtus d'une identité précolombienne à l'aune des connaissances de la fin du XIX ${ }^{e}$ siècle.
Pascal Mongne, From Golgotha to tzompantli : the "Aztec" crystal skulls - Throughout the world there are several dozen of these rock-crystal skulls, often attributed to the Aztec. They include twelve larger models, which have become famous thanks to their supposed "eschatolotogical" powers and have inspired a recent film.

New studies have, however, shown that these remarkable pieces are not Aztec in origin and were, in fact, crafted at a much later date using quartz from outside Mexico. As their authenticity is in doubt, does it then follow that we should consider them to be fakes, designed to deceive? The possibility of erroneous attribution merits attention and suggests that they might have been objects of catholic devotion, such as crucifix bases, dismantled, forgotten then rediscovered and finally re-covered with a preColombian identity in line with late-nineteenth century scientific knowledge. 